OPEN

SUBJECT AREAS:

WEIGHT MANAGEMENT

LIPIDOMICS

NUTRITION

Received

11 July 2014

Accepted

26 November 2014

Published

5 January 2015

Correspondence and requests for materials should be addressed to

S.S. (s.santosa@

concordia.ca)

* These authors contributed equally to this work.

\section{Effects of weight loss via high fat vs. low fat alternate day fasting diets on free fatty acid profiles}

\author{
Krista A. Varady ${ }^{1 *}$, Vi T. Dam ${ }^{2,3 *}$, Monica C. Klempel ${ }^{3}$, Matthew Horne ${ }^{2,3}$, Rani Cruz ${ }^{2,3}$, \\ Cynthia M. Kroeger ${ }^{1} \&$ Sylvia Santosa ${ }^{2,3}$
}

\begin{abstract}
'Department of Kinesiology and Nutrition, University of Illinois at Urbana-Champaign, Chicago, IL, USA, ${ }^{2}$ Department of Exercise Science, Concordia University, Montreal, QC, Canada, ${ }^{3}$ Nutrition, Obesity, and Metabolism Lab, PERFORM Centre, Concordia University, Montreal, QC, Canada.
\end{abstract}

Cardiovascular disease risk is associated with excess body weight and elevated plasma free fatty acid (FFA) concentrations. This study examines how an alternate-day fasting (ADF) diet high (HF) or low (LF) in fat affects plasma FFA profiles in the context of weight loss, and changes in body composition and lipid profiles. After a 2-week weight maintenance period, 29 women (BMI 30-39.9 kg/m ${ }^{2}$ ) 25-65 years old were randomized to an 8-week ADF-HF ( $45 \%$ fat) diet or an ADF-LF ( $25 \%$ fat) diet with $25 \%$ energy intake on fast days and ad libitum intake on feed days. Body weight, BMI and waist circumference were assessed weekly and body composition was measured using dual $\mathrm{x}$-ray absorptiometry (DXA). Total and individual FFA and plasma lipid concentrations were measured before and after weight loss. Body weight, BMI, fat mass, total cholesterol, LDL-C and triglyceride concentrations decreased $(\mathrm{P}<0.05)$ in both groups. Total FFA concentrations also decreased $(P<0.001)$. In the ADF-LF group, decreases were found in several more FFAs than in the ADF-HF group. In the ADF-HF group, FFA concentrations were positively correlated with waist circumference. Depending on the macronutrient composition of a diet, weight loss with an ADF diet decreases FFA concentrations through potentially different mechanisms.

R isk of cardiovascular disease (CVD) has been associated with elevated plasma free fatty acid (FFA) concentrations ${ }^{1,2}$. Higher FFA concentrations in plasma have been associated with atherosclerosis, thrombosis and hypertension ${ }^{1,3,4}$. Moreover, fatty acid composition has been shown to affect disease risk. For example, high proportions of palmitic acid $(16: 0)$ have been associated with higher triglycerides, low density lipoprotein cholesterol (LDL-C), and total cholesterol ${ }^{5,6}$. Both expansion of adipose tissue and reduced FFA clearance contribute to elevated FFA concentrations commonly seen in obesity ${ }^{7}$.

Weight loss through caloric restriction has been found to reduce many of the risk factors of CVD ${ }^{8-10}$. However, the effects of weight loss on plasma FFA concentrations are unclear. Some studies have found no differences ${ }^{11,12}$, while others have observed decreases ${ }^{13,14}$ in FFA concentrations as a result of weight loss. The varied results of weight loss on FFA concentrations may depend on the dietary protocol and macronutrient distribution.

Alternate day fasting (ADF) has been shown to be effective for weight loss. ADF regimens include alternating days of food consumption (feed days) with fast days where food intake is restricted to $25 \%$ of a person's energy needs in a day. Weight loss via ADF in adults has resulted in several cardioprotective changes in the blood lipid profile including lower LDL cholesterol, and increased average LDL particle size ${ }^{15-17}$. Whether these ADFassociated changes in weight loss extend to changes in plasma total FFA and FFA composition is unknown. Additionally, we do not know how the macronutrient composition of ADF diets impact the FFA profile. The objective of this study is to examine how ADF diets of different macronutrient compositions affect plasma FFA profiles in the context of weight loss, and changes in body composition and lipid profiles.

\section{Results}

Subjects. Of the 35 women who started the study, 29 completed the trial. One subject was excluded due to scheduling conflicts and five subjects were excluded due to not having lost any weight during the weight loss period or noncompliance to the diet regime ( $\mathrm{n}=2$ in the ADF-HF group and $\mathrm{n}=3$ in the ADF-LF group). Baseline characteristics of the ADF-HF and ADF-LF groups are reported in Table 1. The groups were well matched for age, BMI and body composition. 
Table 1 | Participant characteristics before (week 3) and after (week 10) the weight loss period

\begin{tabular}{|c|c|c|c|c|}
\hline$\frac{\text { Group }}{\text { Time }}$ & \multicolumn{2}{|c|}{ ADF-LF $(n=15)$} & \multicolumn{2}{|c|}{ ADF-HF $(n=14)$} \\
\hline Age (y) & \multicolumn{2}{|c|}{$43.2 \pm 2.3$} & \multicolumn{2}{|c|}{$42.4 \pm 3.0$} \\
\hline Body mass index $\left(\mathrm{kg} / \mathrm{m}^{2}\right)$ & $34.4 \pm 0.8$ & $32.7 \pm 0.7^{a}$ & $34.6 \pm 0.7$ & $32.8 \pm 0.7^{a}$ \\
\hline$\%$ Fat & $41.6 \pm 1.1$ & $41.8 \pm 1.1$ & $40.4 \pm 0.8$ & $39.2 \pm 1.0$ \\
\hline Fat mass $(\mathrm{kg})$ & $36.9 \pm 1.9$ & $35.4 \pm 1.9^{a}$ & $36.1 \pm 1.4$ & $33.2 \pm 1.5^{\mathrm{a}}$ \\
\hline LDL cholesterol (mmol/L) & $3.0 \pm 0.2$ & $2.3 \pm 0.2^{a}$ & $2.8 \pm 0.2$ & $2.3 \pm 0.2^{a}$ \\
\hline HDL cholesterol (mmol/L) & $1.5 \pm 0.1$ & $1.5 \pm 0.1$ & $1.6 \pm 0.1$ & $1.6 \pm 0.1$ \\
\hline Triglycerides (mmol/L) & $1.1 \pm 0.1$ & $0.9 \pm 0.1^{a}$ & $1.4 \pm 0.2$ & $1.3 \pm 0.2^{a}$ \\
\hline Glucose (mg/dL) & $107.4 \pm 2.9$ & $105.1 \pm 2.0$ & $108.4 \pm 3.0$ & $105.7 \pm 2.2$ \\
\hline
\end{tabular}

Values reported as mean \pm SEM. Alternate day fasting high fat diet (ADF-HF); alternate day fasting low fat diet (ADF-LF).

ap $<0.05$ within group difference (from week 3 to week 10).

Weight loss, body composition and plasma lipid concentrations. During the initial weight maintenance period (week 1-2), no betweengroup differences were found in any of the baseline characteristics. Over the 8-week ADF diet period, both the ADF-HF and the ADF-LF groups decreased $(\mathrm{P}<0.05)$ in weight, $\mathrm{BMI}$, fat mass, and waist circumference WC (Table 1). There were no differences in the extent of weight loss, changes in BMI, and WC between groups. There was a trend $(\mathrm{P}=0.09)$ for a group*time interaction in fat mass. Total cholesterol, LDL-C, and triglycerides decreased in both groups $(\mathrm{P}<0.05)$ during the weight loss period, whereas HDL-C did not change over time. There was a trend $(\mathrm{P}=0.08)$ for a group*time interaction in LDL-C whereby a larger decrease in LDL-C was observed in the ADF-LF vs. the ADF-HF group.

FFA concentrations. With weight loss, total FFAs decreased $(\mathrm{P}<$ $0.001)$ in the ADF-LF group by an average of $98.62 \pm 3.66 \mu \mathrm{mol} / \mathrm{L}$. A trend was found $(\mathrm{P}=0.05)$ in the ADF-HF group, where total FFA decreased by $53.93 \pm 3.73 \mu \mathrm{mol} / \mathrm{L}$ (Figure 1). Changes in total FFA concentrations did not differ between groups. Significant decreases were observed in LNA $(\mathrm{P}=0.01)$, MA $(\mathrm{P}=0.049)$, AA $(\mathrm{P}=0.007)$, LA $(\mathrm{P}=0.004), \mathrm{PA}(\mathrm{P}=0.001)$ and $\mathrm{OA}(\mathrm{P}=0.001)$ in the ADF-LF group (Table 2 ). In contrast, in the ADF-HF group there were significant reductions in FFA concentrations of LNA $(P=0.02)$ and LA $(P=0.048)$.

Relationship between changes in body composition and FFA concentrations. Overall, a decrease in weight correlated with a decrease in MA $(r=0.391 ; P=0.039)$. In the ADF-LF group weight loss correlated with decreases in DHA $(\mathrm{r}=0.642 ; \mathrm{P}=$ 0.013). In the ADF-HF group, lower fat mass correlated with decreases in LA $(\mathrm{r}=0.635 ; \mathrm{P}=0.015)$. Changes in waist circumference in the ADF-HF group correlated with changes in LNA $(r=0.554 ; \mathrm{P}=0.040), \mathrm{LA}(\mathrm{r}=0.700 ; \mathrm{P}=0.005)$, PA $(\mathrm{r}=$ $0.623 ; \mathrm{P}=0.017)$, OA $(\mathrm{r}=0.610 ; \mathrm{P}=0.027)$, and total FFA $(\mathrm{r}=$ $0.623 ; \mathrm{P}=0.017$ ) (Figure 2). No correlation was found between waist circumference and any FFA in the ADF-LF group.

Relationship between changes in FFA and lipid concentrations. In the ADF-HF group, changes in LNA concentrations negatively correlated with total cholesterol $(\mathrm{r}=-0.601 ; \mathrm{P}=0.023)$ and HDL $(\mathrm{r}=-0.548 ; \mathrm{P}=0.042)$. In the ADF-HF group, DHA negatively correlated $(\mathrm{r}=-0.573 ; \mathrm{P}=0.032)$ with LDL-C. In the ADF-LF group, LA was positively correlated $(\mathrm{r}=0.508$; $\mathrm{P}=0.044)$ with HDL-C.

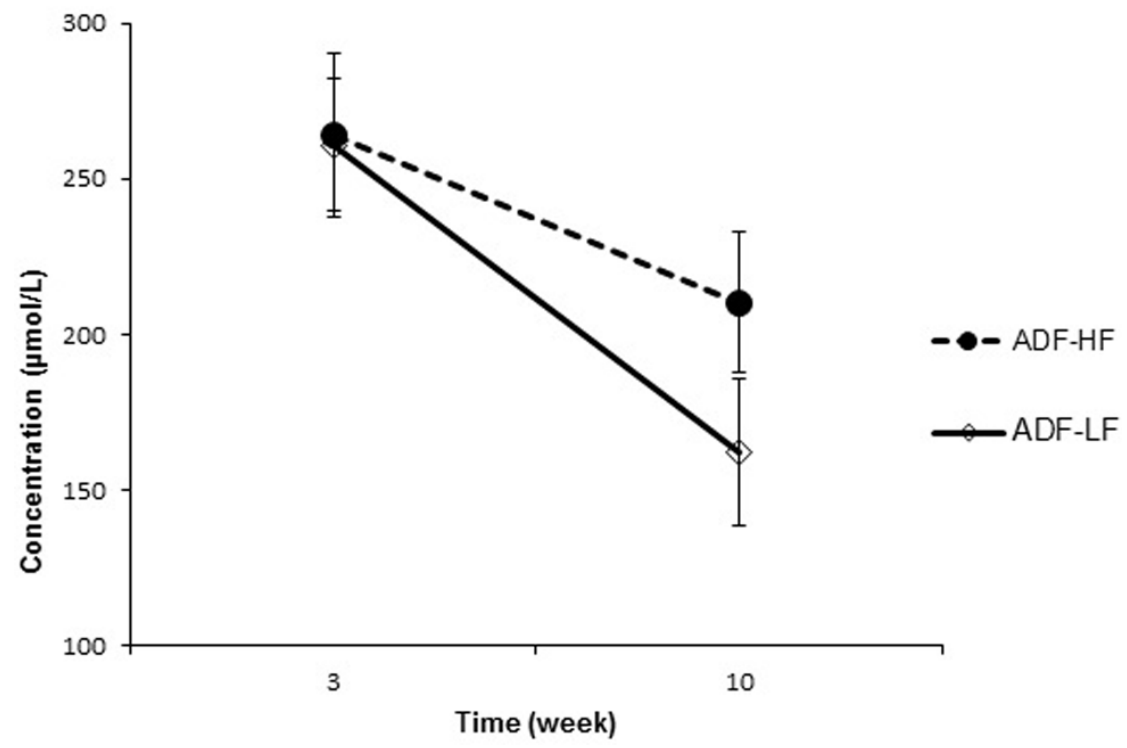

Figure 1 Total FFAs in ADF groups from week 3 to week 10. Mean total fatty acid concentrations in ADF-HF and ADF-LF groups for weeks 3 and 10 of the study. Values reported as mean \pm SEM. Alternate day fasting high fat diet (ADF-HF); alternate day fasting low fat diet (ADF-LF). 
Table 2 | FFA changes during an ADF-LF and ADF-HF weight loss diet

\begin{tabular}{|c|c|c|c|c|}
\hline FFA ( $\mu \mathrm{mol} / \mathrm{L})$ & \multicolumn{2}{|c|}{ ADF-LF } & \multicolumn{2}{|c|}{ ADF-HF } \\
\hline LNA & $11.08 \pm 0.31$ & $10.96 \pm 0.22^{a}$ & $12.07 \pm 0.38$ & $11.59 \pm 0.29^{a}$ \\
\hline MA & $19.79 \pm 0.51$ & $19.15 \pm 0.46^{a}$ & $20.72 \pm 0.46$ & $20.14 \pm 0.48$ \\
\hline $\mathrm{AA}$ & $11.88 \pm 0.38$ & $11.34 \pm 0.34^{a}$ & $12.67 \pm 0.42$ & $12.50 \pm 0.28$ \\
\hline LA & $79.92 \pm 2.98$ & $75.02 \pm 3.47^{a}$ & $89.39 \pm 5.42$ & $85.10 \pm 3.38^{a}$ \\
\hline Total FFA & $260.89 \pm 22.69$ & $162.27 \pm 24.35^{b}$ & $264.14 \pm 27.89$ & $210.21 \pm 24.16$ \\
\hline
\end{tabular}

\section{Discussion}

Elevated total and individual plasma FFA concentrations observed in obesity have been linked with cardiovascular disease risk $^{1,2,5}$. During weight loss, diet composition may affect FFA concentrations ${ }^{6,7}$. Our study examined the effect of weight loss with a high fat ADF diet on total and individual FFA concentrations compared to a low fat ADF diet. We found that after weight loss, both groups had decreases in total FFA concentrations. However, even though weight loss and regional body composition results were similar between the two groups, almost all FFAs in the ADF-LF group decreased whereas changes were only noted in LA and LNA in the ADF-HF group. Despite similar changes in waist circumference, decreases in WC were correlated with decreases in several individual FFAs in the ADF-HF group only.

In our study, we evaluated circulating concentrations of FFA. Elevated total and individual plasma FFA concentrations observed in obesity have been linked with cardiovascular disease risk and atherosclerosis ${ }^{1,5,18,19}$. FFAs has been observed to impair vasodilation in blood vessels through activating apoptosis in the endothelium ${ }^{20}$. Furthermore, FFA is the main energy source for the myocardium; high levels of FFA play a role in damaging the myocardium, exacerbating heart failure and leading to myocardial infarction ${ }^{21}$. Thus far, no ADF studies have analysed circulating FFA profiles in order to determine whether any cardio-protective changes occur at the FFA level. The results of our FFA analysis show that weight loss with an ADF diet decreases total FFA concentrations, and that both the ADFLF and ADF-HF diets could be effective at reducing FFA concentrations. Though no significant group*time interactions were observed, the decrease in total FFA concentrations with an ADF-LF diet was almost 2 times that of the ADF-HF diet. Previously, effects of weight loss on FFA concentrations appear to be variable with some studies showing decreases in FFA ${ }^{11,13,14}$, while others show no change $e^{22-24}$ or even increase ${ }^{25-27}$ in FFA. Some of these studies achieved weight loss using a very low calorie diet, surgery or hypocaloric diets with varying macronutrient composition. Therefore, the dietary regimen used in these studies during the weight loss period may be a source of variability in changes of FFA to weight loss.

Individual FFA concentrations have been shown to contribute to CVD risk differently. Thrombosis and arrhythmia have been associated with a number of saturated FFA including palmitic acid ${ }^{28,29}$. Palmitic, oleic, and myristic acid have been positively associated with lipid risk factors of $\mathrm{CVD}^{6}$. Our study also showed that LA, DHA and LNA were associated with lipid risk factors of CVD. Patients postmyocardial infarction were also shown to have greater palmitic and oleic acid and lower linoleic acid compared to age-matched controls ${ }^{30}$. With an ADF-LF diet, we observed a significant reduction in more types of FFAs (LNA, MA, AA, LA, PA and OA) vs. in the
ADF-HF group (LNA and LA). This suggests that the low fat ADF diet was more effective at decreasing FFA concentrations vs. the high fat ADF diet. To our knowledge this is the first study to examine changes in individual FFA composition in the context of a weight loss program using ADF with varying macronutrient composition. Normal weight participants who consumed a high fat diet also observed changes in the proportion of LA and LNA compared with a low fat $\operatorname{diet}^{31}$. These results suggest that LA and LNA are more sensitive to changes with the consumption of a high fat diet. Therefore, combining a high fat diet with weight loss via an ADF protocol would likely have a greater effect on these FFAs, as observed in this study.

There were strong correlations in the ADF-HF group where decreases in waist circumference were associated with decreases in several FFAs. In contrast, there were no such correlations between waist circumference and FFAs in the ADF-LF group. Waist circumference is considered a surrogate marker of abdominal adiposity and visceral fat mass ${ }^{32}$. We hypothesize that in weight loss, the levels of dietary fat intake and changes in abdominal fat have a collective effect on decreases in FFA concentrations. The relatively larger contribution of a diet low in fat to changes in FFA concentrations may mask the relationship between abdominal fat mass and FFA concentrations. In contrast, weight loss with a high fat diet may have a smaller effect in lowering FFA concentrations in comparison to the greater effect of decreases in abdominal fat. Regardless of the relative contribution of dietary fat content on FFA lowering, changes in abdominal fat mass during weight loss with an ADF diet resulted in cardioprotective changes in FFA concentrations.

A major strength of the study was that both the ADF-LF and ADFHF group were matched for body composition. Additionally, body composition changed equally in the ADF-LF and ADF-HF groups. Thus, the effects of a HF and LF diet on FFA concentrations could be examined without the confounding effects of changes in body composition. Additionally, since all the meals were provided and the ratio of SFA:MUFA:PUFA was similar in the high and low fat diets, the effects of overall fat content on FFA concentrations could be isolated.

Due to a communication error during sample processing, hormonal regulators of lipolysis were not measured. While insulin inhibits FFA release, other hormones such as epinephrine stimulate FFA release over a wide range ${ }^{33,34}$. Thus, we are not able to determine the mechanisms that underlie the changes we saw in FFA composition as a result of a ADF-HF or ADF-LF diet. Another limitation of this study was that both intervention groups lost weight during the weight maintenance period. However, there were no between group differences in weight changes. It is conceivable that decreases in weight during the weight maintenance period diluted observable decreases in FFA concentrations during the weight loss that followed. 
a
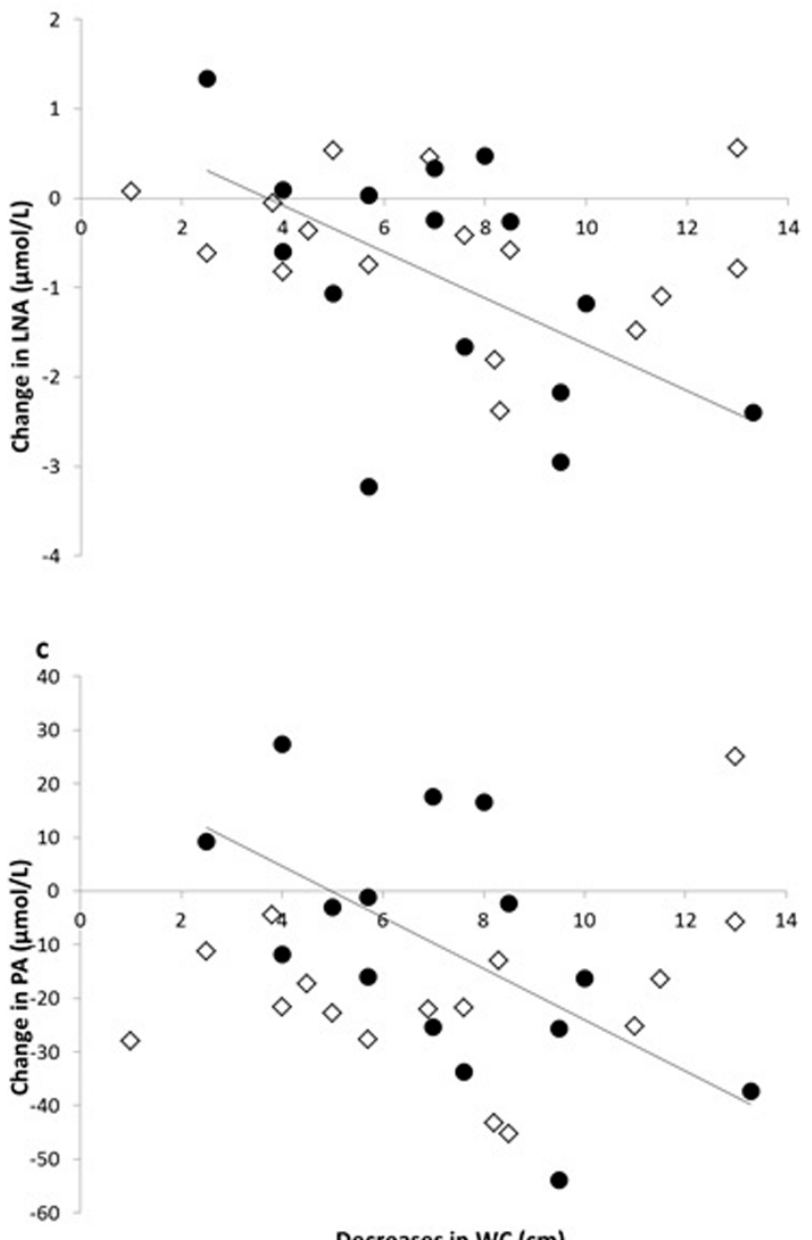

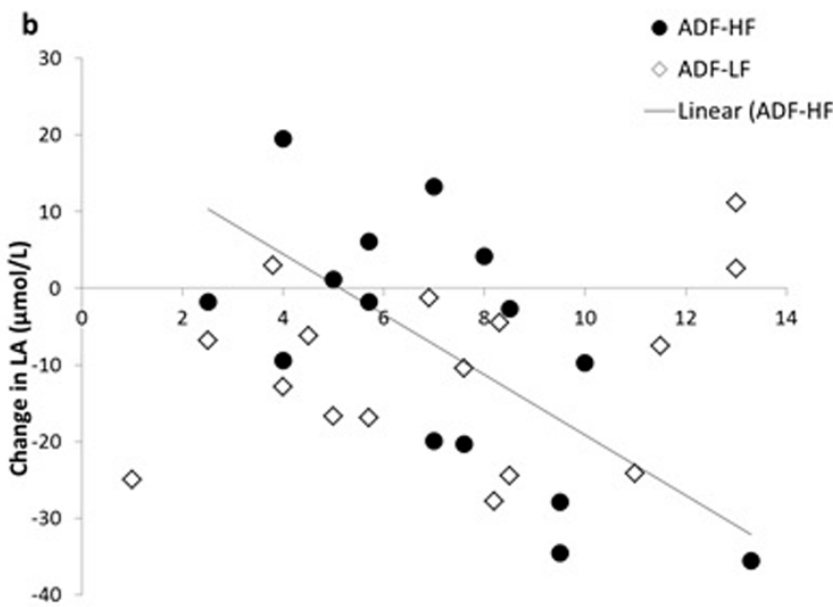

d

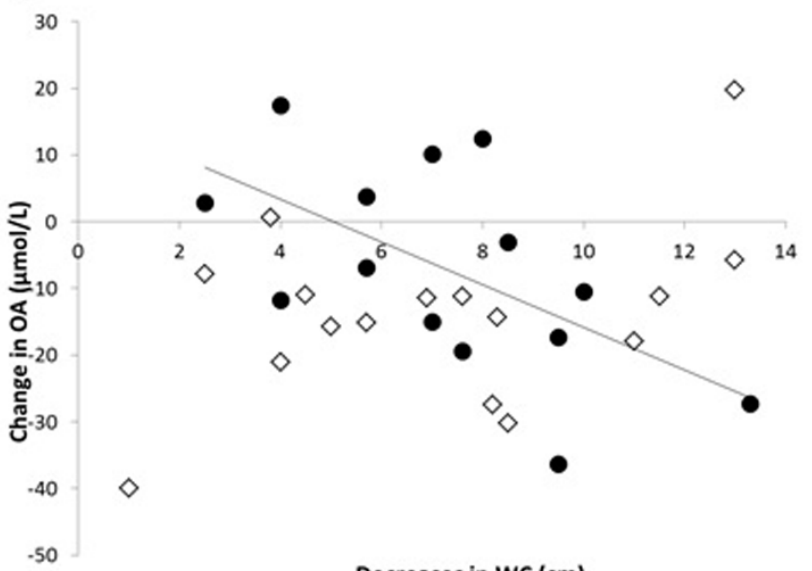

Decreases in WC $(\mathrm{cm})$

Figure $2 \mid$ Relationship between decreases in waist circumference (WC) and changes in $\alpha$-linolenic acid (LNA; Panel a), linoleic acid (LA; Panel b), palmitic acid (PA; Panel c), and oleic acid (OA; Panel b). Each panel shows a significant relationship $(\mathrm{P}<0.05)$ between changes in WC and the FFA with an ADF-HF diet. There were no relationships between changes in WC and FFAs with an ADF-LF diet.

As we observed significant changes in FFA concentrations during the weight loss period, the effect of the initial weight lost during the weight maintenance period on FFA concentrations was likely to be minimal.

In summary, our findings are the first to demonstrate that a weight loss intervention with ADF-HF or ADF-LF can reduce total and individual plasma FFA concentrations though it appears that a LF diet may be more effective at lowering FFA. Though both diets equally reduced waist circumference, we found several strong correlations between WC and changes in individual FFAs in the ADF-HF group that were not present in the ADF-LF group. Since there were no differences in changes in FFA composition between groups, the correlations between WC and FFAs found only in the ADF-HF group imply that ADF-HF and ADF-LF diets may lower FFA concentrations through different mechanisms. Overall, the decreases in FFA concentrations after ADF weight loss may be cardio-protective. Future studies might include individual FFA profiles when evaluating macronutrient composition, as the effects of specific FFAs in this context are not well known.

\section{Methods}

Subjects. Participants were recruited from the Chicago area and were randomized by stratified random sampling based on BMI and age. Subjects were then randomized to a ADF-HF or ADF-LF group, as previously described ${ }^{35}$. Briefly, potential participants were eligible if they were female, 25 to 65 years of age, with a body mass index (BMI) between 30 and $39.9 \mathrm{~kg} / \mathrm{m}^{2}$. Participants had to be weight stable for 3 months prior to the beginning of the study, sedentary or lightly active, non-smokers, non-diabetic, and not taking any medications. Postmenopausal women were included provided they maintained a stable dose of their current hormone replacement therapy. All volunteers provided written informed consent. All study protocols were carried our in accordance with guidelines approved by the Office for the Protection of Research Subjects at the University of Illinois at Chicago, Chicago, USA and the Human Research Ethics Committee at Concordia University, Montreal, Canada.

Diet. Food provided was prepared in the metabolic kitchen of the Human Nutrition Research Unit at the University of Illinois, Chicago. All subjects underwent a 2-week high fat or low fat weight maintenance period (week 1-2), followed by an 8-week ADF weight-loss diet (week 3-10). The ADF-HF group received $45 \%$ fat ( $14 \%$ saturated fat, $20 \%$ monounsaturated fat, $11 \%$ polyunsaturated fat) and the ADF-LF group received $25 \%$ fat $(6 \%$ saturated fat, $13 \%$ monounsaturated, $6 \%$ polyunsaturated fat). A detailed description of the diet composition and energy requirements is provided elsewhere ${ }^{35}$. Subjects were provided with 3 meals on each feed day and one meal on each fast day. All subjects were asked to consume their meals at the same time each day.

Weight loss and body composition. Body weight measurements were taken weekly following an overnight fast (to the nearest $0.5 \mathrm{~kg}$ ) in light clothing and without shoes using a balance beam scale (HealthOMeter; Sunbeam Products, Boca Raton, FL). Height was measured using a wall-mounted stadiometer (to the nearest $0.1 \mathrm{~cm}$ ). BMI was assessed as $\mathrm{kg} / \mathrm{m}^{2}$. Total fat mass and fat-free mass was determined by dual energy X-ray absorptiometry (DXA) (QDR 4500 W, Hologic Inc. Arlington, MA) at baseline, week 3 and week 10 . Waist circumference was taken by a flexible measuring tape (to the nearest $0.1 \mathrm{~cm}$ ), midway between the lower costal margin and super iliac crest during a period of expiration. Participants wore a pedometer (Digi-Walker SW-200, Yamax Corp., Japan) during the study to ensure maintenance of regular physical activity habits. 
Plasma lipid and free fatty acid analysis. Blood samples were collected in the morning after an overnight fast at baseline, week 3 and week 10. Exercise, alcohol, and coffee were restricted for $24 \mathrm{~h}$ before each visit. Blood was centrifuged for $10 \mathrm{~min}$ at $520 \mathrm{G}$ in $4^{\circ} \mathrm{C}$ and plasma was stored at $-80^{\circ} \mathrm{C}$ until analyzed. Plasma lipid samples were analysed as previously described ${ }^{35}$. FFA were quantified via ultra performance liquid chromatography (UPLC) (Waters ACUITY UPLC, Milford, Massachusetts). $\alpha$-linolenate (LNA), docosahexanoate (DHA), arachadonate (AA), and linoleate (LA) standards were purchased from Sigma-Aldrich. Myristate (MA), palmitate (PA) and oleate $(\mathrm{OA})$ standards were purchased from Nu-Check prep. All standards were $>99 \%$ pure. FFA standards were dissolved in $4 \%$ fatty acid free albumin phosphate buffer. The individual standard solutions were combined to form an external mixed FFA standard. D31 palmitate (Cambridge Isotope Laboratories, Inc., Tewksbury, Massachusetts) was used as an internal standard and added to plasma samples and external standards. Plasma samples and the mixed FFA standards were extracted and derivatized as previously described ${ }^{36}$. Samples were then transferred to a UPLC vial in 20 : $80 \mathrm{H} 2 \mathrm{O}$ :acetonitrile solution and $10 \mu \mathrm{L}$ were injected onto a $55^{\circ} \mathrm{C}$ heated Acquity UPLC BEH C18 $1.7 \mu \mathrm{M} 2.1 \times 150 \mu \mathrm{mm}$ column eluted with H2O:acetonitrile 20:80 at a $\mathrm{mL} / \mathrm{min}$ rate. Peaks were detected with a photodiode array (PDA) detector at $242 \mathrm{~nm}$

Statistical methods. All data was analyzed using SPSS software (version 20.0, SPSS Inc., Chicago, IL). Results are presented as mean \pm SEM. Normality was assessed by Shapiro-Wilks tests. Independent samples t-tests were used to test baseline differences between groups. Repeated-measures ANOVA were used to assess for significant differences with time as the within-subject factor and diet as the betweensubject factor. Post-hoc analyses were done using a Tukey's test. Pearson correlations were performed to assess the relationships between body composition and FFA concentrations. Differences were considered significant at $\mathrm{P}<0.05$.

1. Pilz, S. et al. Elevated plasma free fatty acids predict sudden cardiac death: a 6.85 year follow-up of 3315 patients after coronary angiography. Eur Heart J 28, 2763-2769, doi:10.1093/eurheartj/ehm343 (2007).

2. Boden, G., Chen, X., Ruiz, J., White, J. V. \& Rossetti, L. Mechanisms of fatty acidinduced inhibition of glucose uptake. J Clin Invest 93, 2438-2446, doi:10.1172/ JCI117252 (1994).

3. Breitling, L. P., Rothenbacher, D., Grandi, N. C., Marz, W. \& Brenner, H. Prognostic usefulness of free fatty acids in patients with stable coronary heart disease. Am J Cardiol 108, 508-513, doi:10.1016/j.amjcard.2011.03.076 (2011)

4. Boden, G. Interaction between free fatty acids and glucose metabolism. Curr Opin Clin Nutr Metab Care 5, 545-549 (2002).

5. Paillard, F. et al. Plasma palmitoleic acid, a product of stearoyl-coA desaturase activity, is an independent marker of triglyceridemia and abdominal adiposity. Nutr Metab Cardiovasc Dis 18, 436-440, doi:10.1016/j.numecd.2007.02.017 (2008).

6. Sabin, M. A. et al. Fasting nonesterified fatty acid profiles in childhood and their relationship with adiposity, insulin sensitivity, and lipid levels. Pediatrics 120, e1426-1433, doi:10.1542/peds.2007-0189 (2007).

7. Boden, G. Obesity and free fatty acids. Endocrinol Metab Clin North Am 37, 635-646, viii-ix, doi:10.1016/j.ecl.2008.06.007 (2008).

8. Bhutani, S., Klempel, M. C., Berger, R. A. \& Varady, K. A. Improvements in coronary heart disease risk indicators by alternate-day fasting involve adipose tissue modulations. Obesity 18, 2152-2159, doi:10.1038/oby.2010.54 (2010).

9. Canoy, D. Coronary heart disease and body fat distribution. Curr Atheroscler Rep 12, 125-133, doi:10.1007/s11883-010-0092-9 (2010).

10. Lavie, C. J., Milani, R. V. \& Ventura, H. O. Obesity and cardiovascular disease: risk factor, paradox, and impact of weight loss. J Am Coll Cardiol 53, 1925-1932, doi:10.1016/j.jacc.2008.12.068 (2009).

11. Keogh, J. B., Luscombe-Marsh, N. D., Noakes, M., Wittert, G. A. \& Clifton, P. M. Long-term weight maintenance and cardiovascular risk factors are not different following weight loss on carbohydrate-restricted diets high in either monounsaturated fat or protein in obese hyperinsulinaemic men and women. $\mathrm{Br}$ Nutr 97, 405-410, doi:10.1017/S0007114507252687 (2007).

12. Faraj, M., Jones, P., Sniderman, A. D. \& Cianflone, K. Enhanced dietary fat clearance in postobese women. J Lipid Res 42, 571-580 (2001).

13. Astrup, A. et al. Impaired glucose-induced thermogenesis and arterial norepinephrine response persist after weight reduction in obese humans. Am J Clin Nutr 51, 331-337 (1990).

14. Chearskul, S., Delbridge, E., Shulkes, A., Proietto, J. \& Kriketos, A. Effect of weight loss and ketosis on postprandial cholecystokinin and free fatty acid concentrations. Am J Clin Nutr 87, 1238-1246 (2008).

15. Varady, K. A., Bhutani, S., Church, E. C. \& Klempel, M. C. Short-term modified alternate-day fasting: a novel dietary strategy for weight loss and cardioprotection in obese adults. Am J Clin Nutr 90, 1138-1143, doi:10.3945/ajcn.2009.28380 (2009)

16. Johnson, J. B. et al. Alternate day calorie restriction improves clinical findings and reduces markers of oxidative stress and inflammation in overweight adults with moderate asthma. Free Radic Biol Med 42, 665-674, doi:10.1016/ j.freeradbiomed.2006.12.005 (2007).
17. Eshghinia, S. \& Mohammadzadeh, F. The effects of modified alternate-day fasting diet on weight loss and CAD risk factors in overweight and obese women. J Diabetes Metab Disord 12, 4, doi:10.1186/2251-6581-12-4 (2013).

18. Havmoeller, R. et al. Elevated plasma free fatty acids are associated with sudden death: a prospective community-based evaluation at the time of cardiac arrest. Heart Rhythm 11, 691-696, doi:10.1016/j.hrthm.2014.01.012 (2014).

19. Stich, V. \& Berlan, M. Physiological regulation of NEFA availability: lipolysis pathway. Proc Nutr Soc 63, 369-374, doi:10.1079/PNS2004350 (2004).

20. Hufnagel, B. et al. Unsaturated fatty acids isolated from human lipoproteins activate protein phosphatase type 2Cbeta and induce apoptosis in endothelial cells. Atherosclerosis 180, 245-254, doi:10.1016/j.atherosclerosis.2004.12.021 (2005).

21. Pilz, S. et al. Free fatty acids are independently associated with all-cause and cardiovascular mortality in subjects with coronary artery disease. J Clin Endocrinol Metab 91, 2542-2547, doi:10.1210/jc.2006-0195 (2006).

22. Heilbronn, L. K., Noakes, M. \& Clifton, P. M. Effect of energy restriction, weight loss, and diet composition on plasma lipids and glucose in patients with type 2 diabetes. Diabetes Care 22, 889-895 (1999).

23. Rabol, R. et al. Reduced skeletal muscle mitochondrial respiration and improved glucose metabolism in nondiabetic obese women during a very low calorie dietary intervention leading to rapid weight loss. Metabolism 58, 1145-1152, doi:10.1016/ j.metabol.2009.03.014 (2009).

24. McLaughlin, T., Abbasi, F., Lamendola, C., Kim, H. S. \& Reaven, G. M. Metabolic changes following sibutramine-assisted weight loss in obese individuals: role of plasma free fatty acids in the insulin resistance of obesity. Metabolism 50, 819-824, doi:10.1053/meta.2001.24220 (2001)

25. Hernandez, T. L. et al. Lack of suppression of circulating free fatty acids and hypercholesterolemia during weight loss on a high-fat, low-carbohydrate diet. Am J Clin Nutr 91, 578-585, doi:10.3945/ajcn.2009.27909 (2010).

26. Bryson, J. M. et al. Changes in glucose and lipid metabolism following weight loss produced by a very low calorie diet in obese subjects. Int J Obes Relat Metab Disord 20, 338-345 (1996)

27. Kanaley, J. A., Cryer, P. E. \& Jensen, M. D. Fatty acid kinetic responses to exercise. Effects of obesity, body fat distribution, and energy-restricted diet. J Clin Invest 92, 255-261, doi:10.1172/JCI116559 (1993).

28. Frayn, K. N., Williams, C. M. \& Arner, P. Are increased plasma non-esterified fatty acid concentrations a risk marker for coronary heart disease and other chronic diseases? Clin Sci (London) 90, 243-253 (1996).

29. Makiguchi, M., Kawaguchi, H., Tamura, M. \& Yasuda, H. Effect of palmitic acid and fatty acid binding protein on ventricular fibrillation threshold in the perfused rat heart. Cardiovasc Drugs Ther 5, 753-761 (1991).

30. Lemieux, S., Prud'homme, D., Bouchard, C., Tremblay, A. \& Despres, J. P. Sex differences in the relation of visceral adipose tissue accumulation to total body fatness. Am J Clin Nutr 58, 463-467 (1993).

31. Raatz, S. K., Bibus, D., Thomas, W. \& Kris-Etherton, P. Total fat intake modifies plasma fatty acid composition in humans. J Nutr 131, 231-234 (2001).

32. Pouliot, M. C. et al. Waist circumference and abdominal sagittal diameter: best simple anthropometric indexes of abdominal visceral adipose tissue accumulation and related cardiovascular risk in men and women. Am J Cardiol 73, 460-468 (1994).

33. Jensen, M. D., Haymond, M. W., Gerich, J. E., Cryer, P. E. \& Miles, J. M. Lipolysis during fasting. Decreased suppression by insulin and increased stimulation by epinephrine. J Clin Invest 79, 207-213, doi:10.1172/JCI112785 (1987).

34. Jensen, M. D., Caruso, M., Heiling, V. \& Miles, J. M. Insulin regulation of lipolysis in nondiabetic and IDDM subjects. Diabetes 38, 1595-1601 (1989).

35. Klempel, M. C., Kroeger, C. M. \& Varady, K. A. Alternate day fasting (ADF) with a high-fat diet produces similar weight loss and cardio-protection as ADF with a low-fat diet. Metabolism 62, 137-143, doi:10.1016/j.metabol.2012.07.002 (2013)

36. Miles, J., Glasscock, R., Aikens, J., Gerich, J. \& Haymond, M. A microfluorometric method for the determination of free fatty acids in plasma. J Lipid Res 24, 96-99 (1983).

\section{Author contributions}

K.A.V. assisted with the design of the experiment and drafted/revised the manuscript. V.T.D. analyzed and interpreted the data, and drafted/revised the manuscript. M.C.K. conceived and designed the experiment, conducted the clinical trial, analyzed the data and was the study manager. M.H. and R.C. generated, collected, analyzed the data and revised the manuscript. C.M.K. assisted with the collection of study data. S.S. obtained funding for the analyses, assisted with the design of the experiment, analyzed the data, and drafted/ revised the manuscript. All authors read and approved the final manuscript.

\section{Additional information}

Funding source PERFORM Seed Funding, Concordia University, NSERC Discovery Grant, University of Illinois at Chicago, Department of Kinesiology and Nutrition, Departmental funding. SS is also the recipient of a Canada Research Chair, Tier II in Clinical Nutrition. Funding sources were not involved in the study design, collection, and analysis/ interpretation of data in the writing of the manuscript or in the decision to submit the article for publication.

Competing financial interests: The authors declare no competing financial interests. 
How to cite this article: Varady, K.A. et al. Effects of weight loss via high fat vs low fat alternate day fasting diets on free fatty acid profiles. Sci. Rep. 5, 7561; DOI:10.1038/ srep07561 (2015)

(c) (i) (2) This work is licensed under a Creative Commons Attribution-NonCommercial-

ShareAlike 4.0 International License. The images or other third party material in this article are included in the article's Creative Commons license, unless indicated otherwise in the credit line; if the material is not included under the Creative Commons license, users will need to obtain permission from the license holder in order to reproduce the material. To view a copy of this license, visit http:// creativecommons.org/licenses/by-nc-sa/4.0/ 


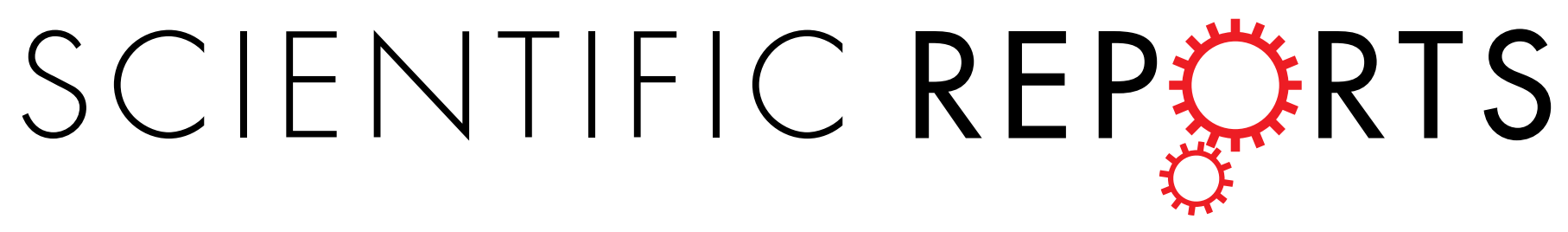

\section{Corrigendum: Effects of weight loss via high fat vs. low fat alternate day fasting diets on free fatty acid profiles}

Krista A. Varady, Vi T. Dam, Monica C. Klempel, Matthew Horne, Rani Cruz, Cynthia M. Kroeger \& Sylvia Santosa

Scientific Reports 5:7561; doi: 10.1038/srep07561; published online 05 January 2015; updated on 05 August 2015 The Competing financial interests statement in this Article should read:

Krista Varady is an author of the book, "The Every Other Day Diet", which is a guide to alternate day fasting for the general public. 